\title{
Macrolide-Resistant Mycoplasma pneumoniae Infection, Japan, 2008-2015
}

\author{
Takaaki Tanaka, Tomohiro Oishi, Ippei Miyata, \\ Shoko Wakabayashi, Mina Kono, Sahoko Ono, \\ Atsushi Kato, Yoko Fukuda, Aki Saito, \\ Eisuke Kondo, Hideto Teranishi, Yuhei Tanaka, \\ Tokio Wakabayashi, Hiroto Akaike, Satoko Ogita, \\ Naoki Ohno, Takashi Nakano, \\ Kihei Terada, Kazunobu Ouchi
}

We evaluated isolates obtained from children with Mycoplasma pneumoniae infection throughout Japan during 20082015. The highest prevalence of macrolide-resistant $M$. pneumoniae was $81.6 \%$ in 2012 , followed by $59.3 \%$ in 2014 and $43.6 \%$ in 2015 . The prevalence of macrolide-resistant M. pneumoniae among children in Japan has decreased.

Since the early 2000s, macrolide-resistant Mycoplasma pneumoniae isolates have been identified in Japan. We previously reported the results of our national surveillance study that investigated the prevalence of macrolide-resistant $M$. pneumoniae infection among children in Japan during 2008-2012 (1). An M. pneumoniae pandemic occurred in Japan during 2010-2012, especially among children. Similar pandemics also occurred in other countries (2). However, the prevalence of macrolide-resistant M. pneumoniae infection also gradually increased at the same time in Asia, including Japan $(1,2)$. We investigated the prevalence of macrolide-resistant $M$. pneumoniae infection after the pandemic.

\section{The Study}

Children with respiratory tract infections who visited 68 medical institutions in Japan were classified according to district: 1) Kyushu (population 14 million); 2) ChugokuShikoku (11 million); 3) Kinki (20 million); 4) KantoChubu (62 million); and 5) Tohoku-Hokkaido (14 million) (Figure). Most patients had been enrolled in the Atypical Pathogen Study Group before the 2010-2012 pandemic; however, some did not participate in our previous study reported in 2013 (1). Here, we report the data for January 2008-December 2015.

As previously reported (1), pediatricians collected nasopharyngeal swab samples and sputum samples, when available, from children with respiratory tract infections. Participants' parents provided informed consent before

Author affiliation: Kawasaki Medical School, Okayama, Japan

DOI: https://doi.org/10.3201/eid2310.170106 sample collection. The Ethics Committee of the Kawasaki Medical School (Kurashiki, Japan) approved the study protocol on December 8, 2014 (no. 286-3).

Nasopharyngeal swab specimens were collected with a sterile swab (Nippon Menbo, Saitama, Japan). After collection, each swab was placed into $3 \mathrm{~mL}$ of Universal Vial Transport Medium (Becton, Dickinson and Company, Sparks, MD, USA) and transported at room temperature within 2 days to Kawasaki Medical School by a parcel delivery system. Crude DNA extracts were obtained with the following procedure: $300 \mu \mathrm{L}$ of resuspended transport medium was transferred into a $1.5-\mathrm{mL}$ microtube centrifuged at $4{ }^{\circ} \mathrm{C}, 20,000 \times \mathrm{g}$ for $30 \mathrm{~min}$, after which $285 \mu \mathrm{L}$ of supernatant was discarded; the remainder was transferred into a thin-wall $200-\mu \mathrm{L}$ PCR tube after resuspension with $85 \mu \mathrm{L}$ lysis buffer by gentle pipetting. This suspension was incubated at $55^{\circ} \mathrm{C}$ for $60 \mathrm{~min}$, followed by $100^{\circ} \mathrm{C}$ for $10 \mathrm{~min}$ before cooling to $4^{\circ} \mathrm{C}$. The composition of the lysis buffer was Tris- $\mathrm{HCl}(\mathrm{pH} 8.3) 2 \mathrm{mmol} / \mathrm{L}, \mathrm{KCl} 10 \mathrm{mM}, \mathrm{MgCl}_{2}$ $0.045 \mathrm{mM}$, Triton X-100 0.45\%, Tween $200.45 \%$, and RNA-grade Proteinase K (Thermo Fisher Scientific Inc., Waltham, MA, USA) $0.4 \mu \mathrm{g} / \mu \mathrm{L}$. M. pneumoniae DNA was detected by real-time PCR targeting a conserved part of the gene coding for the P1 adhesin (3).

We searched for mutations at sites 2063, 2064, and 2617 in domain V of 23S rRNA of $M$. pneumoniae using a direct sequencing method with isolates or samples with a positive PCR result, as reported previously (3). For this study, we investigated 1,448 samples obtained from patients in Japan who had respiratory tract infections; we detected M. pneumoniae DNA by real-time PCR and searched for mutations using a direct sequencing method.

The overall prevalence rate of macrolide-resistant $M$. pneumoniae in Japan was $70.2 \%(1,016 / 1,448)$ and ranged from $43.7 \%$ in Kyushu to $89.3 \%$ in Kanto-Chubu (Table 1). When divided into 3 time periods (prepandemic, pandemic, and postpandemic), the overall rate of macrolide-resistant M. pneumonia was substantially lower in the post epidemic period than in the pandemic period; macrolide-resistant $M$. pneumoniae isolates decreased in 4 of the 5 locations.

The peak rate of macrolide-resistant $M$. pneumoniae infection was $81.6 \%$ in 2012 (493/604) (Table 2). Rates of macrolide-resistant $M$. pneumoniae infection gradually decreased as follows: $65.8 \%(25 / 38)$ in $2013,59.3 \%$ $(16 / 27)$ in 2014 , and $43.6 \%(65 / 149)$ in 2015 . The most frequent mutation was A2063G mutation (95.8\%), followed 


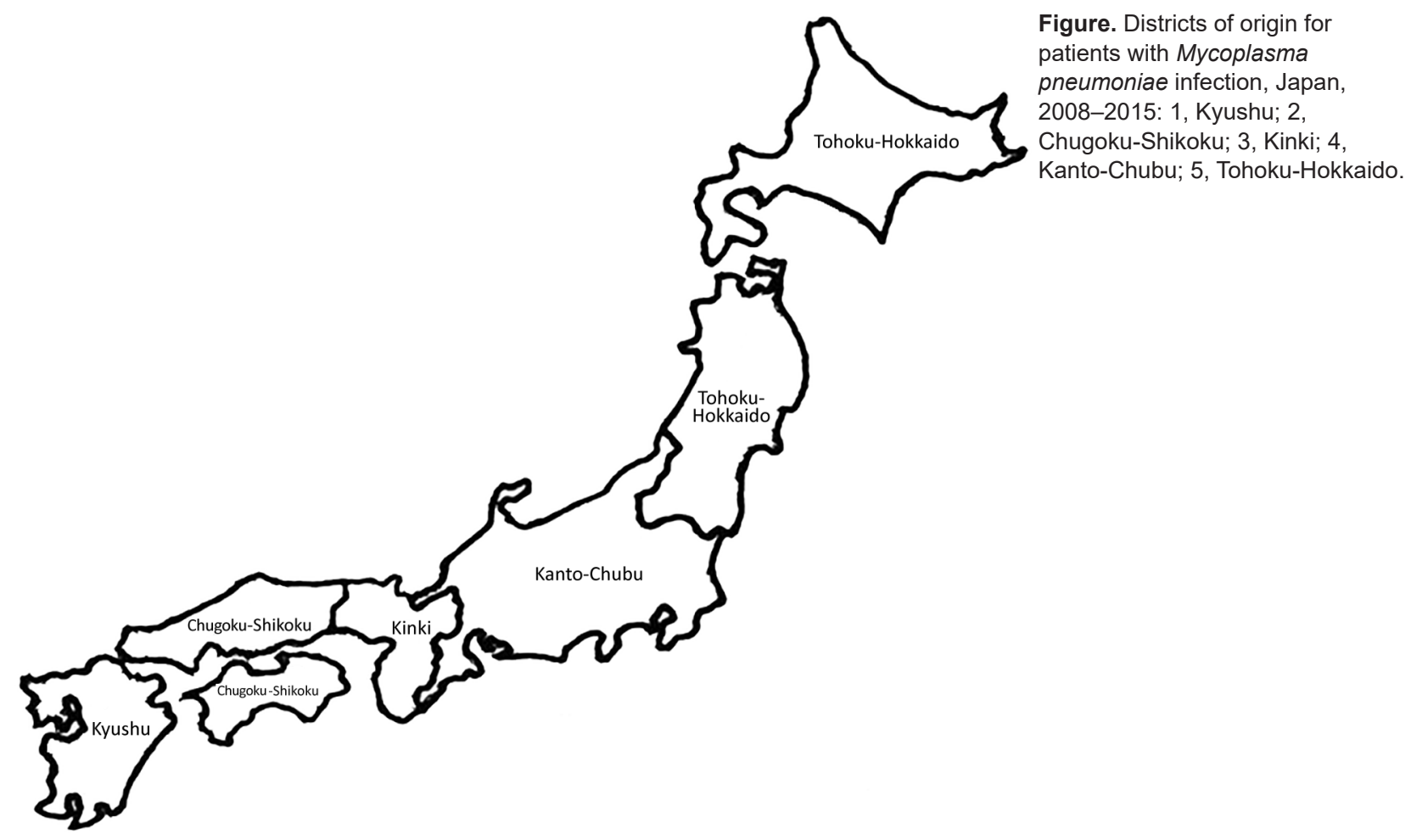

by A2063T (3.1\%), A2064G (0.6\%), A2063C (0.3\%), C2617G (0.2\%), and C2617T (0.1\%).

\section{Conclusions}

The prevalence of macrolide-resistant $M$. pneumoniae infection was high during 2008-2012 but gradually decreased throughout Japan during 2013-2015. One reason for this decrease might be the 2011 publication of guidelines for treating M. pneumoniae pneumonia (4). Because of the higher prevalence of macrolide-resistant M. pneumoniae infection in children than in adults (5), respiratory fluoroquinolone/tosufloxacin was recommended for use in patients in whom $M$. pneumoniae pneumonia responded poorly to macrolide treatment in these guidelines. After the pandemic, the guideline committee addressed the concerns of further accumulation of macrolide-resistant M. pneumoniae in children resulting from constant macrolide use and teeth damage from tetracycline use in children $<8$ years of age (4). Tosufloxacin was approved for use in children in 2010 in Japan and has been used to treat M. pneumoniae infection. Because the guidelines recommend tosufloxacin as a second-line drug, pediatricians in Japan may be using the appropriate antimicrobial drugs for M. pneumoniae infection in accordance with these guidelines, which might have led to the decrease in incidence. Unfortunately, to our knowledge, no reports have been published to support these hypotheses. However, prescriptions for oral antimicrobial drugs in Japan comprise most of the prescriptions for antimicrobial drugs ( 6 ); therefore, we believe that the rate of macrolide-resistant $M$. pneumoniae might be affected by changes in the use of oral macrolide agents.

In addition, the prevalence of macrolide-resistant $M$. pneumoniae infection varies among countries: for example, $13.2 \%$ in the United States (7), 8.3\% in France (8), and $3.1 \%$ in Germany (9). These variations might be attributed to differences in the number of prescription macrolide agents among countries. Although accurately assuming the number of prescription macrolide agents in each country is

\begin{tabular}{|c|c|c|c|c|c|c|}
\hline \multirow[b]{2}{*}{ District } & \multirow{2}{*}{$\begin{array}{c}\text { Average age, y } \\
\text { (range) }\end{array}$} & \multirow{2}{*}{$\begin{array}{l}\text { No. patients } \\
(\mathrm{M}: \mathrm{F})\end{array}$} & \multicolumn{4}{|c|}{ Macrolide resistance, $\%$ (no. positive/total no. patients) ${ }^{*}$} \\
\hline & & & $2008-2010$ & $2011-2012$ & $2013-2015$ & Total \\
\hline Kyushu & $6.4(0-14)$ & $239(1.2: 1)$ & - & $64.1(82 / 128)$ & $27.9(31 / 111)$ & $47.3(113 / 239)$ \\
\hline Chugoku-Shikoku & $7.3(0-15)$ & $623(1.4: 1)$ & $68.6(59 / 86)$ & $70.6(339 / 480)$ & $80.7(46 / 57)$ & $71.3(444 / 623)$ \\
\hline Kinki & $7.1(1-15)$ & $227(1: 1)$ & $33.3(7 / 21)$ & $86.1(162 / 188)$ & $66.7(12 / 18)$ & $79.7(181 / 227)$ \\
\hline Kanto-Chubu & $7.5(0-13)$ & $268(1.1: 1)$ & - & $80.1(197 / 246)$ & $72.7(16 / 22)$ & $79.5(213 / 268)$ \\
\hline Hokkaido-Tohoku & $7.7(0-13)$ & $91(1.2: 1)$ & $84.2(32 / 38)$ & $68.1(32 / 47)$ & $16.7(1 / 6)$ & $71.4(65 / 91)$ \\
\hline Total & $7.3(0-15)$ & $1,448(1.3: 1)$ & $67.6(98 / 145)$ & $74.6(812 / 1,089)$ & $49.5(106 / 214)$ & $70.2(1,016 / 1,448)$ \\
\hline
\end{tabular}

*2008-2010, prepandemic; 2011-2012, pandemic; 2013-2015, postpandemic. 
Table 2. Rates of macrolide-resistant Mycoplasma pneumoniae and point mutations in domain $\mathrm{V}$ of 23S rRNA, Japan

\begin{tabular}{lccccccccc}
\hline $\begin{array}{l}\text { Variable } \\
\text { Macrolide resistance, }\end{array}$ & 2008 & 2009 & 2010 & 2011 & 2012 & 2013 & 2014 & 2015 & Total \\
$\begin{array}{l}\text { \% (no. positive/total } \\
\text { no. patients) }\end{array}$ & $(10 / 18)$ & $\begin{array}{c}72.7 \\
(8 / 11)\end{array}$ & $\begin{array}{c}69.0 \\
(80 / 116)\end{array}$ & $\begin{array}{c}65.8 \\
(319 / 485)\end{array}$ & $\begin{array}{c}81.6 \\
(493 / 604)\end{array}$ & $\begin{array}{c}65.8 \\
(25 / 38)\end{array}$ & $\begin{array}{c}59.3 \\
(16 / 27)\end{array}$ & $\begin{array}{c}43.6 \\
(65 / 149)\end{array}$ & $\begin{array}{c}70.2 \\
(1,016 /\end{array}$ \\
\hline $\begin{array}{l}\text { Point mutations, no. (\%) } \\
\text { A2063G }\end{array}$ & $10(100)$ & $8(100)$ & $80(100)$ & $301(94.4)$ & $471(95.5)$ & $25(100)$ & $16(100)$ & $62(95.2)$ & $97.3(95.8)$ \\
A2063C & 0 & 0 & 0 & $3(0.9)$ & 0 & 0 & 0 & 0 & $3(0.3)$ \\
A2063T & 0 & 0 & 0 & $15(4.7)$ & $14(2.3)$ & 0 & 0 & $2(3.2)$ & $31(3.1)$ \\
A2064G & 0 & 0 & 0 & 0 & $6(1.0)$ & 0 & 0 & 0 & $6(0.6)$ \\
C2617G & 0 & 0 & 0 & 0 & $2(0.3)$ & 0 & 0 & 0 & $2(0.2)$ \\
C2617T & 0 & 0 & 0 & 0 & 0 & 0 & 0 & $1(1.6)$ & $1(0.1)$ \\
\hline
\end{tabular}

difficult, we can estimate the macrolide resistance rate of Streptococcus pneumoniae among those countries. A recent report supported the hypothesis that antimicrobial selection pressure results in clonal expansion of existing resistant strains (10). In Japan and China, which have a high prevalence of macrolide-resistant $M$. pneumoniae $(3,5)$, the rates of macrolide-resistant $S$. pneumoniae also are very high $(11,12)$. In countries with low rates of macrolide-resistant M. pneumoniae, such as the United States, France, and Germany $(7,8,9)$, the prevalence rates of macrolide-resistant $S$. pneumoniae are low $(13,14)$. However, the mechanisms of macrolide resistance are difficult to compare between Streptococcus and Mycoplasma (15). Therefore, we also interviewed the pediatricians who collected the samples for this study, and analysis of their responses is ongoing. Upon completion of these interviews, we will be able to report patients' background characteristics, such as previous use of macrolides and medical examination histories; this information may provide further insight into the decreased prevalence of macrolide-resistant M. pneumoniae infection.

In summary, the prevalence of macrolide-resistant $M$. pneumoniae infection in children in Japan was high and increased between 2008 and 2012 but declined thereafter. Careful continuous monitoring of macrolide-resistant $M$. pneumoniae infection rates in Japan and other countries is needed.

\section{Acknowledgments}

We thank Reiji Kimura and Mika Suwa for their technical assistance and all the clinicians who participated by taking samples in the Atypical Pathogen Study Group.

Dr. Tanaka is a senior lecturer in the Department of Pediatrics at Kawasaki Medical School. His primary research interest is vaccines for travelers.

\section{References}

1. Kawai Y, Miyashita N, Kubo M, Akaike H, Kato A, Nishizawa Y, et al. Nationwide surveillance of macrolide-resistant Mycoplasma pneumoniae infection in pediatric patients. Antimicrob Agents Chemother. 2013;57:4046-9. http://dx.doi.org/10.1128/ AAC.00663-13

2. Zhao F, Liu G, Wu J, Cao B, Tao X, He L, et al. Surveillance of macrolide-resistant Mycoplasma pneumoniae in Beijing, China, from 2008 to 2012. Antimicrob Agents Chemother. 2013; 57:1521-3. http://dx.doi.org/10.1128/AAC.02060-12

3. Miyashita N, Kawai Y, Yamaguchi T, Ouchi K, Oka M; Atypical Pathogen Study Group. Clinical potential of diagnostic methods for the rapid diagnosis of Mycoplasma pneumoniae pneumonia in adults. Eur J Clin Microbiol Infect Dis. 2011;30:439-46. http://dx.doi.org/10.1007/s10096-010-1107-8

4. Joint Committee of Japanese Society of Pediatric Infectious Disease and Japanese Society of Pediatric Pulmonology. Pneumonia. In: Ouchi K, Kurosaki T, Okada K, editors. Guidelines for management of respiratory infectious diseases in children in Japan 2011 [in Japanese]. Tokyo: Kyowa Kikaku Ltd.; 2011.p. 44

5. Miyashita N, Kawai Y, Akaike H, Ouchi K, Hayashi T, Kurihara T, et al.; Atypical Pathogen Study Group. Macrolideresistant Mycoplasma pneumoniae in adolescents with community-acquired pneumonia. BMC Infect Dis. 2012;12:126. http://dx.doi.org/10.1186/1471-2334-12-126

6. Muraki Y, Yagi T, Tsuji Y, Nishimura N, Tanabe M, Niwa T, et al. Japanese antimicrobial consumption surveillance: first report on oral and parenteral antimicrobial consumption in Japan (2009-2013). J Glob Antimicrob Resist. 2016;7:19-23. http://dx.doi.org/10.1016/j.jgar.2016.07.002

7. Zheng X, Lee S, Selvarangan R, Qin X, Tang YW, Stiles J, et al. Macrolide-resistant Mycoplasma pneumoniae, United States. Emerg Infect Dis. 2015;21:1470-2. http://dx.doi.org/10.3201/ eid2108.150273

8. Pereyre S, Touati A, Petitjean-Lecherbonnier J, Charron A, Vabret A, Bébéar C. The increased incidence of Mycoplasma pneumoniae in France in 2011 was polyclonal, mainly involving M. pneumoniae type 1 strains. Clin Microbiol Infect.2013;19:E212-7. http://dx.doi.org/10.1111/ 1469-0691.12107

9. Dumke R, Schnee C, Pletz MW, Rupp J, Jacobs E, Sachse K, et al.; Capnetz Study Group. Mycoplasma pneumoniae and Chlamydia spp. infection in community-acquired pneumonia, Germany, 2011-2012. Emerg Infect Dis. 2015;21:426-34. http://dx.doi.org/10.3201/eid2103.140927

10. Keenan JD, Klugman KP, McGee L, Vidal JE, Chochua S, Hawkins P, et al. Evidence for clonal expansion after antibiotic selection pressure: pneumococcal multilocus sequence types before and after mass azithromycin treatments. J Infect Dis. 2015;211:988-94. http://dx.doi.org/10.1093/infdis/jiu552

11. Okade H, Funatsu T, Eto M, Furuya Y, Mizunaga S, Nomura N, et al. Impact of the pneumococcal conjugate vaccine on serotype distribution and susceptibility trends of pediatric non-invasive Streptococcus pneumoniae isolates in Tokai, Japan over a 5-year period. J Infect Chemother. 2014;20:423-8. http://dx.doi.org/10.1016/j.jiac.2014.03.010

12. Liu WK, Liu Q, Chen DH, Liang HX, Chen XK, Chen MX, et al. Epidemiology of acute respiratory infections in children in Guangzhou: a three-year study. PLoS One. 2014;9:e96674. http://dx.doi.org/10.1371/journal.pone.0096674 
13. Jenkins SG, Farrell DJ. Increase in pneumococcus macrolide resistance, United States. Emerg Infect Dis. 2009;15:1260-4. http://dx.doi.org/10.3201/eid1508.081187

14. Farrell DJ, Couturier C, Hryniewicz W. Distribution and antibacterial susceptibility of macrolide resistance genotypes in Streptococcus pneumoniae: PROTEKT year 5 (2003-2004). Int J Antimicrob Agents. 2008;31:245-9. http://dx.doi.org/ 10.1016/j.ijantimicag.2007.10.022

15. Bley $C$, van der Linden $M$, Reinert RR. $\operatorname{mef}(A)$ is the predominant macrolide resistance determinant in Streptococcus pneumoniae and Streptococcus pyogenes in Germany. Int J Antimicrob Agents. 2011;37:425-31. http://dx.doi.org/10.1016/ j.ijantimicag.2011.01.019

Address for correspondence: Tomohiro Oishi, Kawasaki Medical School—Pediatrics, 577, Matsushima, Kurashiki Kurashiki Okayama 701-0192, Japan; email: oo0612@med.kawasaki-m.ac.jp

\section{September 2016: Antimicrobial Resistance}

- Co-Infections in Visceral Pentastomiasis, Democratic Republic of the Congo

- Multistate US Outbreak of Rapidly Growing Mycobacterial Infections Associated with Medical Tourism to the Dominican Republic, 2013-2014

- Virulence and Evolution of West Nile Virus, Australia, 1960-2012

- Phylogeographic Evidence for 2 Genetically Distinct Zoonotic Plasmodium knowlesi Parasites, Malaysia

- Hemolysis after Oral Artemisinin Combination Therapy for Uncomplicated Plasmodium falciparum Malaria

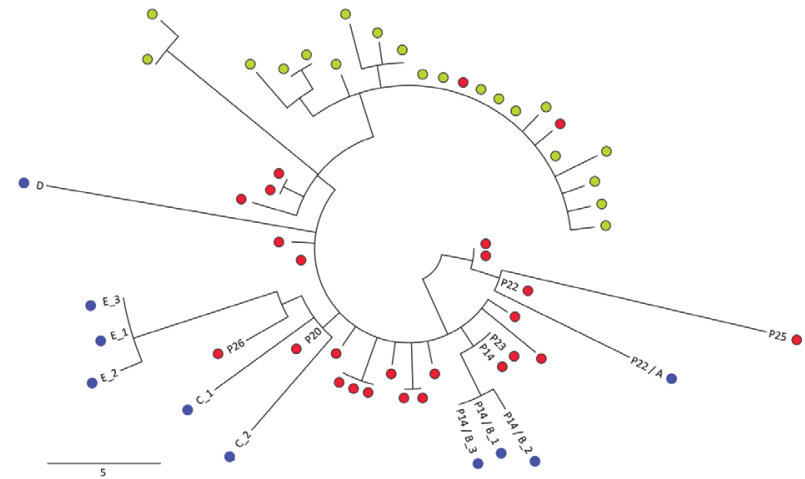

- Enterovirus D68 Infection in Children with Acute Flaccid Myelitis, Colorado, USA, 2014

- Middle East Respiratory Syndrome Coronavirus Transmission in Extended Family, Saudi Arabia, 2014

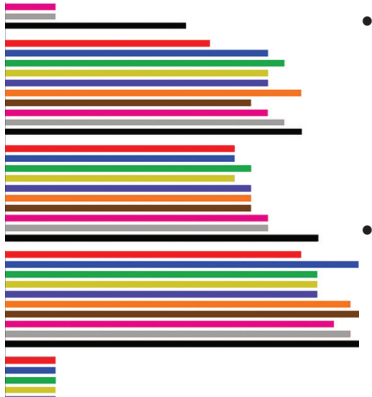

- Exposure-Specific and AgeSpecific Attack Rates for Ebola Virus Disease in EbolaAffected Households, Sierra Leone

- Outbreak of Achromobacter xylosoxidans and Ochrobactrum anthropi Infections after Prostate Biopsies, France, 2014
- Human Babesiosis, Bolivia, 2013

- Assessment of Community Event-Based Surveillance for Ebola Virus Disease, Sierra Leone, 2015

- Probable Rabies Virus Transmission through Organ Transplantation, China, 2015

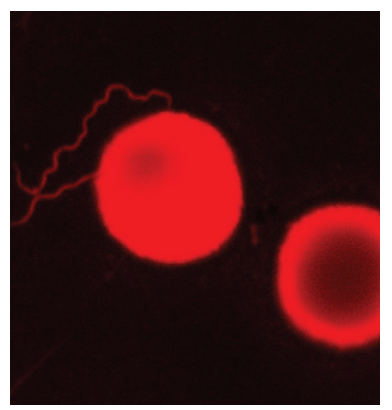

- Cutaneous Melioidosis Cluster Caused by Contaminated Wound Irrigation Fluid

- Possible Role of Fish and Frogs as Paratenic Hosts of Dracunculus medinensis, Chad

- Time Lags between Exanthematous Illness Attributed to Zika Virus, Guillain-Barré Syndrome, and Microcephaly, Salvador, Brazil

- Use of Unamplified RNA/cDNA-Hybrid Nanopore Sequencing for Rapid Detection and Characterization of RNA Viruses

- Importation of Hybrid Human-Associated Trypanosoma cruzi Strains of Southern South American Origin, Colombia

- Lyssavirus in Indian Flying Foxes, Sri Lanka

- Survival and Growth of Orientia tsutsugamushi in Conventional Hemocultures

- Chagas Disease Screening in Maternal Donors of Publicly Banked Umbilical Cord Blood, United States

- Multilocus Sequence Typing Tool for Cyclospora cayetanensis

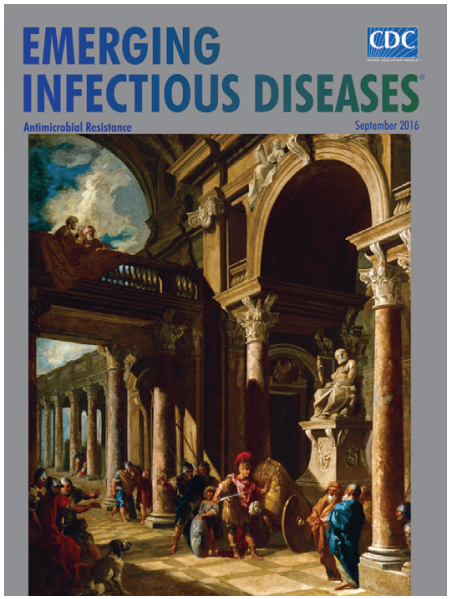

